\title{
Weighted Composition Operators between Weighted Bergman Spaces and Weighted Banach Spaces of Holomorphic Functions
}

\author{
Elke WOLF
}

\author{
Mathematical Institute \\ University of Paderborn \\ D-33095 Paderborn — Germany \\ lichte@math.uni-paderborn.de
}

Received: July 2, 2007

Accepted: December 13, 2007

\begin{abstract}
We characterize boundedness and compactness of weighted composition operators acting between weighted Bergman spaces $A_{w, p}$ and weighted Banach spaces $H_{v}^{\infty}$ of holomorphic functions.

Key words: weighted Bergman space, weighted composition operator, weighted Banach space of holomorphic functions.

2000 Mathematics Subject Classification: 47B33, 47B38.
\end{abstract}

\section{Introduction}

Let $v$ and $w$ be strictly positive bounded continuous functions (weights) on the open unit disk $D$ in the complex plane and $H(D)$ be the space of holomorphic functions. We are interested in operators acting between the weighted Bergman space

$$
A_{w, p}=\left\{f \in H(D) ;\|f\|_{w, p}:=\left(\int_{D}|f(z)|^{p} w(z) d A(z)\right)^{\frac{1}{p}}<\infty\right\}, \quad 1 \leq p<\infty,
$$

where $d A(z)$ is the area measure on $D$ normalized so that area of $D$ is 1 and the weighted Banach space of holomorphic functions (weighted Bergman space of infinite order)

$$
H_{v}^{\infty}:=\left\{f \in H(D) ;\|f\|_{v}:=\sup _{z \in D} v(z)|f(z)|<\infty\right\} .
$$


Let $\phi: D \rightarrow D$ and $\psi: D \rightarrow \mathbb{C}$ be analytic mappings. Such maps induce a linear weighted composition operator $\psi C_{\phi}(f)=\psi(f \circ \phi)$ between spaces of holomorphic functions of the type defined above.

Composition operators and weighted composition operators have been studied on various spaces of holomorphic functions, see, e.g., $[2-5,10]$. For more general information on composition operators we refer to the monographs $[6,9]$. In this article we want to characterize boundedness and compactness of weighted composition operators acting between spaces of the type defined above in terms of the weights. Our result is similar to the one Sharma and Sharma obtained in [10] for standard weights.

\section{Preliminaries}

We denote by $B_{v}^{\infty}$ the closed unit ball of $H_{v}^{\infty}$. The so-called associated weights are an important tool to handle weighted spaces of holomorphic functions. For a weight $v$ the associated weight $\tilde{v}$ is defined as follows

$$
\tilde{v}(z):=\frac{1}{\sup \left\{|f(z)| ; f \in H_{v}^{\infty},\|f\|_{v} \leq 1\right\}}=\frac{1}{\left\|\delta_{z}\right\|_{H_{v}^{\infty}}}, \quad z \in D,
$$

where $\delta_{z}$ is the point evaluation of $z$. The associated weights are also continuous and $\tilde{v} \geq v>0$ (see [1]). Furthermore, for each $z \in D$ there is $f_{z} \in H_{v}^{\infty},\|f\|_{v} \leq 1$, such that $\left|f_{z}(z)\right|=\frac{1}{\tilde{v}(z)}$. A weight is called essential if there is a constant $C>0$ with

$$
v(z) \leq \tilde{v}(z) \leq C v(z) \text { for every } z \in D .
$$

For examples of essential weights and conditions when weights are essential see $[1-3]$.

We fix $a \in D$ and consider the automorphism $\varphi_{a}(z):=\frac{z-a}{1-\bar{a} z}, z \in D$, which interchanges 0 and $a$. Furthermore we use the fact that

$$
\varphi_{a}^{\prime}(z)=\frac{1-|a|^{2}}{(1-\bar{a} z)^{2}}, \quad z \in D .
$$

\section{Results}

We first need the following auxiliary result. The following lemma is well-known for standard weights (see [7] or [8]) but to the best of our knowledge not known for other weights.

Lemma 2.1. Let $w$ be a weight of the form $w=|u|$ where $u$ is a holomorphic function without any zeros on $D$. Then

$$
|f(z)| \leq \frac{1}{\left(1-|z|^{2}\right)^{\frac{2}{p}} w(z)^{\frac{1}{p}}}\|f\|_{w, p}
$$

for all $z \in D, f \in A_{w, p}$. 
Proof. Let $\alpha \in D$ be an arbitrary point. Consider the map

$$
T_{\alpha}: A_{w, p} \longrightarrow A_{w, p}, \quad T_{\alpha}(f(z))=f\left(\varphi_{\alpha}(z)\right) \varphi_{\alpha}^{\prime}(z)^{\frac{2}{p}} \frac{u\left(\varphi_{\alpha}(z)\right)^{\frac{1}{p}}}{u(z)^{\frac{1}{p}}} .
$$

This map is an isometry since a change of variables yields

$$
\begin{aligned}
\left\|T_{\alpha} f\right\|_{w, p}^{p} & =\int_{D} w(z)\left|f\left(\varphi_{\alpha}(z)\right)\right|^{p}\left|\varphi_{\alpha}^{\prime}(z)\right|^{2} \frac{w\left(\varphi_{\alpha}(z)\right)}{w(z)} d A(z) \\
& =\int_{D}\left|f\left(\varphi_{\alpha}(z)\right)\right|^{p}\left|\varphi_{\alpha}^{\prime}(z)\right|^{2} w\left(\varphi_{\alpha}(z)\right) d A(z) \\
& =\int_{D} w(t)|f(t)|^{p} d A(t)=\|f\|_{w, p}^{p} .
\end{aligned}
$$

Now put $g(z)=T_{\alpha}(f(z))$. By the mean-value property we obtain

$$
w(0)|g(0)|^{p} \leq \int_{D} w(z)|g(z)|^{p} d A(z)=\|g\|_{w, p}^{p}=\|f\|_{w, p}^{p} .
$$

Hence

$$
w(0)|g(0)|^{p}=|f(\alpha)|^{p}\left(1-|\alpha|^{2}\right)^{2} w(\alpha) \leq\|f\|_{w, p}^{p} .
$$

Thus $|f(\alpha)| \leq \frac{\|f\|_{w, p}}{\left(1-|\alpha|^{2}\right)^{\frac{2}{p}} w(\alpha)^{\frac{1}{p}}}$. Since $\alpha$ was arbitrary, the claim follows.

The proof of the following result was inspired by [10].

Theorem 2.2. Let $w$ be a weight of the form $w=|u|$ where $u$ is a holomorphic function without any zeros on $D$. Then the weighted composition operator $\psi C_{\phi}$ : $A_{w, p} \rightarrow H_{v}^{\infty}$ is bounded if and only if

$$
\sup _{z \in D} \frac{v(z)|\psi(z)|}{\left(1-|\phi(z)|^{2}\right)^{\frac{2}{p}} \tilde{w}(\phi(z))^{\frac{1}{p}}}<\infty .
$$

Proof. By [1, Example 1.4] we know that under the given assumptions $w=\tilde{w}$. First suppose that $M=\sup _{z \in D} \frac{v(z)|\psi(z)|}{\left(1-|\phi(z)|^{2}\right)^{\frac{2}{p}} w(\phi(z))^{\frac{1}{p}}}<\infty$. By the lemma we know that

$$
|f(z)| \leq \frac{\|f\|_{w, p}}{\left(1-|z|^{2}\right)^{\frac{2}{p}} w(z)^{\frac{1}{p}}}
$$

for all $z \in D$, independent of $f \in A_{w, p}$. Thus, for $z \in D$, we get

$$
\begin{aligned}
\left\|\psi C_{\phi} f\right\|_{v} & =\sup _{z \in D} v(z)|\psi(z)||f(\phi(z))| \\
& \leq \sup _{z \in D} \frac{v(z)|\psi(z)|}{w(\phi(z))^{\frac{1}{p}}\left(1-|\phi(z)|^{2}\right)^{\frac{2}{p}}}\|f\|_{w, p} .
\end{aligned}
$$


For the converse let $a \in D$ be arbitrary. There exists $f_{a}^{p} \in B_{w}^{\infty}$ such that $\left|f_{a}(a)\right|^{p}=\frac{1}{\tilde{w}(a)}$. Now put $g_{a}(z):=f_{a}(z) \varphi_{a}^{\prime}(z)^{\frac{2}{p}}$. Then a change of variables yields

$$
\begin{aligned}
\left\|g_{a}\right\|^{p} & =\int_{D}\left|g_{a}(z)\right|^{p} w(z) d A(z)=\int_{D}\left|f_{a}(z)\right|^{p}\left|\varphi_{a}^{\prime}(z)\right|^{2} w(z) d A(z) \\
& \leq \sup _{z \in D} w(z)\left|f_{a}(z)\right|^{p} \int_{D}\left|\varphi_{a}^{\prime}(z)\right|^{2} d A(z) \leq \int_{D}\left|\varphi_{a}^{\prime}(z)\right|^{2} d A(z)=\int_{D} d A(t)=1 .
\end{aligned}
$$

Next, we assume that there is a sequence $\left(z_{n}\right)_{n \in \mathbb{N}} \subset D$ such that $\left|\phi\left(z_{n}\right)\right| \rightarrow 1$ and

$$
\frac{\left|\psi\left(z_{n}\right)\right| v\left(z_{n}\right)}{\tilde{w}\left(\phi\left(z_{n}\right)\right)^{\frac{1}{p}}\left(1-\left|\phi\left(z_{n}\right)\right|^{2}\right)^{\frac{2}{p}}} \geq n
$$

for every $n \in \mathbb{N}$. Thus consider now $g_{n}(z):=g_{\phi\left(z_{n}\right)}(z)$ for every $n \in \mathbb{N}$ as defined above. Then we obtain that $\left(g_{n}\right)_{n}$ lies in the closed unit ball of $A_{w, p}$ and

$$
c \geq v\left(z_{n}\right)\left|\psi\left(z_{n}\right)\right|\left|g_{n}\left(\phi\left(z_{n}\right)\right)\right|=\frac{v\left(z_{n}\right)\left|\psi\left(z_{n}\right)\right|}{\tilde{w}\left(\phi\left(z_{n}\right)\right)^{\frac{1}{p}}\left(1-\left|\phi\left(z_{n}\right)\right|^{2}\right)^{\frac{2}{p}}} \geq n
$$

for every $n \in \mathbb{N}$, which is a contradiction.

Examples 2.3.

(i) Consider $p=1, w(z)=|1-z|, \psi(z)=\frac{1-z}{2}=1-\frac{z+1}{2}, v(z)=\left(1-|z|^{2}\right)^{2}$, and $\phi(z)=\frac{z+1}{2}$. By [1, Example 1.4] we know that $w=\tilde{w}$. Then we obtain

$$
\sup _{z \in D} \frac{v(z)|\psi(z)|}{\left(1-|\phi(z)|^{2}\right)^{2} w(\phi(z))}=\sup _{z \in D} \frac{\left(1-|z|^{2}\right)^{2}}{\left(1-\left|\frac{z+1}{2}\right|^{2}\right)^{2}}<\infty \text {. }
$$

Hence the corresponding weighted composition operator is bounded.

(ii) Consider $p=1, w(z)=|1-z|, \psi(z)=\frac{1-z}{2}=1-\frac{z+1}{2}, v(z)=1-|z|^{2}$, and $\phi(z)=\frac{z+1}{2}$. By [1, Example 1.4] we know that $w=\tilde{w}$. Then for $z=r \in \mathbb{R}$ by using the rule of L'Hospital we get

$$
\frac{v(r)|\psi(r)|}{\left(1-|\phi(r)|^{2}\right)^{2} w(\phi(r))}=16 \frac{1-r^{2}}{9-12 r-2 r^{2}+4 r^{3}+r^{4}} \rightarrow \infty \quad \text { if } r \rightarrow 1 .
$$

Hence the operator is not bounded.

The proof of the following result was inspired by [10].

Theorem 2.4. Let $w$ be a weight of the form $w=|u|$ where $u$ is a holomorphic function without any zeros on $D$. Moreover let $\phi: D \rightarrow D$ be analytic with $\|\phi\|=1$ and $\psi \in H_{v}^{\infty}$. Then the weighted composition operator $\psi C_{\phi}: A_{w, p} \rightarrow H_{v}^{\infty}$ is compact if and only if

$$
\lim _{r \rightarrow 1} \sup _{\{z ;|\phi(z)|>r\}} \frac{v(z)|\psi(z)|}{\left(1-|\phi(z)|^{2}\right)^{\frac{2}{p}} \tilde{w}(\phi(z))^{\frac{1}{p}}}=0 .
$$


Proof. First, we assume that $(*)$ holds. Let $\left(f_{n}\right)_{n}$ be a bounded sequence in $A_{w, p}$ that converges to zero uniformly on compact subsets of $D$. Let $M=\sup _{n}\left\|f_{n}\right\|_{w, p}<\infty$. Given $\varepsilon>0$ there is $r>0$ such that if $|\phi(z)|>r$, and then

$$
\frac{v(z)|\psi(z)|}{\left(1-|\phi(z)|^{2}\right)^{\frac{2}{p}} \tilde{w}(\phi(z))^{\frac{1}{p}}}<\varepsilon
$$

By Lemma 2.1 we have

$$
\left|f_{n}(z)\right| \leq \frac{\left\|f_{n}\right\|_{w, p}}{\left(1-|z|^{2}\right)^{\frac{2}{p}} w(z)^{\frac{1}{p}}}
$$

Thus, for $z \in D$, we obtain

$$
v(z)\left|\psi C_{\phi} f_{n}(z)\right|=v(z)|\psi(z)|\left|f_{n}(\phi(z))\right| \leq \frac{v(z)|\psi(z)|}{\left(1-|\phi(z)|^{2}\right)^{\frac{2}{p}} w(\phi(z))^{\frac{1}{p}}}\left\|f_{n}\right\|_{w, p} \leq \varepsilon M
$$

for all $n$.

On the other hand, since $f_{n} \rightarrow 0$ uniformly on $\{u ;|u| \leq r\}$, there is an $n_{0} \in \mathbb{N}$ such that, if $|\phi(z)| \leq r$ and $n \geq n_{0}$, then $\left|f_{n}(\phi(z))\right|<\varepsilon$. By assumption we know $\psi \in H_{v}^{\infty}$. Thus we have $N=\sup _{z \in D} v(z)|\psi(z)|<\infty$ and hence

$$
v(z)\left|\psi C_{\phi} f_{n}(z)\right|=v(z)|\psi(z)|\left|f_{n}(\phi(z))\right| \leq N \varepsilon
$$

Conversely, suppose that $\psi C_{\phi}: A_{w, p} \rightarrow H_{v}^{\infty}$ is compact and that (*) does not hold. Then there are $\delta>0$ and $\left(z_{n}\right)_{n} \subset D$ with $\left|\phi\left(z_{n}\right)\right| \rightarrow 1$ such that

$$
\frac{v\left(z_{n}\right)\left|\psi\left(z_{n}\right)\right|}{w\left(\phi\left(z_{n}\right)\right)^{\frac{1}{p}}\left(1-\left|\phi\left(z_{n}\right)\right|^{2}\right)^{\frac{2}{p}}} \geq \delta .
$$

for all $n$. Since $\left|\phi\left(z_{n}\right)\right| \rightarrow 1$ there exist natural numbers $\alpha(n)$ with $\lim _{n \rightarrow \infty} \alpha(n)=\infty$ and such that $\left|\phi\left(z_{n}\right)\right|^{\alpha(n)} \geq \frac{1}{2}$ for all $n$. For each $n$ consider the function $g_{n}$

$$
g_{n}(z):=f_{\phi_{n}}(z) \varphi_{\phi_{n}}^{\prime}(z)^{\frac{2}{p}} z^{\alpha(n)}
$$

Then $\left(g_{n}\right)_{n}$ is norm bounded and $g_{n} \rightarrow 0$ pointwise because of the factor $z^{\alpha(n)}$. Thus, it follows that a subsequence of $\left(\psi C_{\phi} g_{n}\right)_{n}$ tends to 0 in $H_{v}^{\infty}$. On the other hand

$$
\begin{aligned}
\left\|\psi C_{\phi} g_{n}\right\|_{v} & \geq v\left(z_{n}\right)\left|\psi C_{\phi} g_{n}\left(z_{n}\right)\right|=v\left(z_{n}\right)\left|\psi\left(z_{n}\right)\right|\left|g_{n}\left(\phi\left(z_{n}\right)\right)\right| \\
& =\frac{v\left(z_{n}\right)\left|\psi\left(z_{n}\right)\right|\left|\phi\left(z_{n}\right)\right|^{\alpha(n)}}{\left(1-\left|\phi\left(z_{n}\right)\right|^{2}\right)^{\frac{2}{p}} w\left(\phi\left(z_{n}\right)\right)^{\frac{1}{p}}} \geq \frac{1}{2} \delta
\end{aligned}
$$

which is a contradiction. 


\section{References}

[1] K. D. Bierstedt, J. Bonet, and J. Taskinen, Associated weights and spaces of holomorphic functions, Studia Math. 127 (1998), no. 2, 137-168.

[2] J. Bonet, P. Domański, and M. Lindström, Essential norm and weak compactness of composition operators on weighted Banach spaces of analytic functions, Canad. Math. Bull. 42 (1999), no. 2, 139-148.

[3] J. Bonet, P. Domański, M. Lindström, and J. Taskinen, Composition operators between weighted Banach spaces of analytic functions, J. Austral. Math. Soc. Ser. A 64 (1998), no. 1, 101-118.

[4] J. Bonet, M. Friz, and E. Jordá, Composition operators between weighted inductive limits of spaces of holomorphic functions, Publ. Math. Debrecen 67 (2005), no. 3-4, 333-348.

[5] M. D. Contreras and A. G. Hernandez-Díaz, Weighted composition operators in weighted Banach spaces of analytic functions, J. Austral. Math. Soc. Ser. A 69 (2000), no. 1, 41-60.

[6] C. C. Cowen and B. D. MacCluer, Composition operators on spaces of analytic functions, Studies in Advanced Mathematics, CRC Press, Boca Raton, FL, 1995.

[7] P. Duren and A. Schuster, Bergman spaces, Mathematical Surveys and Monographs, vol. 100, American Mathematical Society, Providence, RI, 2004.

[8] H. Hedenmalm, B. Korenblum, and K. Zhu, Theory of Bergman spaces, Graduate Texts in Mathematics, vol. 199, Springer-Verlag, New York, 2000.

[9] J. H. Shapiro, Composition operators and classical function theory, Universitext: Tracts in Mathematics, Springer-Verlag, New York, 1993.

[10] A. K. Sharma and S. D. Sharma, Weighted composition operators between Bergman-type spaces, Commun. Korean Math. Soc. 21 (2006), no. 3, 465-474. 